OPEN ACCESS

Edited by: Ino Curik,

University of Zagreb, Croatia

Reviewed by:

Dinko Novosel,

University of Zagreb, Croatia

Carlo Fiore Viscomi,

University of Cambridge,

United Kingdom

*Correspondence:

Dexiang Zhang

zhangdexiang0001@sina.com

Xiquan Zhang

xqzhang@scau.edu.cn

${ }^{\dagger}$ These authors have contributed equally to this work

Specialty section:

This article was submitted to

Livestock Genomics,

a section of the journal

Frontiers in Genetics

Received: 16 December 2018 Accepted: 09 December 2019

Published: 17 January 2020

Citation:

Li H, Hu B, Luo Q, Hu S, Luo Y, Zhao B, Gan Y, Li Y, Shi M, Nie Q, Zhang $D$ and Zhang $X$ (2020) Runting and Stunting Syndrome Is Associated With Mitochondrial Dysfunction in Sex-Linked Dwarf Chicken.

Front. Genet. 10:1337. doi: 10.3389/fgene.2019.01337

\section{Runting and Stunting Syndrome Is Associated With Mitochondrial Dysfunction in Sex-Linked Dwarf Chicken}

\author{
Hongmei $\mathrm{Li}^{1,2+}$, Bowen $\mathrm{Hu}^{1,2+}$, Qingbin Luo ${ }^{1,2}$, Shuang Hu ${ }^{1,2}$, Yabiao Luo ${ }^{1,2}$, Bojing Zhao ${ }^{1,2}$, \\ Yanmin Gan ${ }^{1,2}$, Ying Li $^{3}$, Meiqing Shi ${ }^{4}$, Qinghua Nie ${ }^{1,2}$, Dexiang Zhang ${ }^{1,2^{*}}$ and Xiquan Zhang ${ }^{1,2^{*}}$ \\ ${ }^{1}$ Department of Animal Genetics, Breeding and Reproduction, College of Animal Science, South China Agricultural \\ University, Guangzhou, China, ${ }^{2}$ Guangdong Provincial Key Lab of Agro-Animal Genomics and Molecular Breeding and Key \\ Lab of Chicken Genetics, Breeding and Reproduction, Ministry of Agriculture, College of Animal Science, South China \\ Agricultural University, Guangzhou, China, ${ }^{3}$ Institute of Animal Science, Guangdong Academy of Agricultural Sciences, \\ Guangzhou, China, ${ }^{4}$ Division of Immunology, Virginia-Maryland Regional College of Veterinary Medicine, University of \\ Maryland, College Park, College Park, MD, United States
}

Runting and stunting syndrome (RSS) in chicken are commonly known as "frozen chicken." The disease is characterized by lower body weight and slow growth and the incidence rate is widely $5 \%-20 \%$ in sex-linked dwarf (SLD) chickens. However, the etiology of RSS in chickens has plagued researchers for several decades. In this study, histopathology studies demonstrated that the hepatocytes of the RSS chickens contain many mitochondria with damaged and outer and inner membrane along with vacuolar hydropic degeneration. No mtDNA mutation was detected, but our microarray data showed that RSS chickens exhibited abnormal expression of genes, many of which are involved in oxidative phosphorylation (OXPHOS) and fatty acid metabolism. In particular, nuclear gene IGF2BP3 was upregulated in RSS chickens' liver cells. The abnormal expression of these genes is likely to impair the OXPHOS, resulting in reduced ATP synthesis in the hepatocytes of the RSS chickens, which may in turn leads to poor weight gain and retarded growth or stunting of chicks. Our findings suggest that mitochondria dysfunction rather than chronic inflammation is responsible for the reduced growth and RSS in SLD chickens. Mutations in GHR have been shown to compromise mitochondrial function in SLD chickens. Since the mitochondrial damage in the RSS chicken is more severe, we suggest that extra genes are likely to be affected to exacerbate the phenotype.

Keywords: runting and stunting syndrome, chicken, mitochondrial dysfunction, oxidative phosphorylation, ATP synthesis, vacuoles

\section{INTRODUCTION}

Runting and stunting syndrome (RSS) is a condition in which a number of the birds in a flock are considerably smaller owing to delayed growth (Zavala, 2006), and RSS chickens are easily observed in sex-linked dwarf (SLD) chickens. It is known that the SLD chickens are caused by the mutation of growth hormone receptor $(G H R)$ gene and characterized by shorter shanks, and lower body weight 
(Ouyang et al., 2012). But the RSS affected SLD chickens are smaller and lower body weight than SLD chickens. RSS has been reported in poultry flocks around the world, including in the Netherlands (Kouwenhoven et al., 1978) and the UK (Bracewell and Wyeth, 1981). RSS causes economic hardship in the poultry industry through reduced uniformity, increased mortality, decreased body weight, poor feed conversion rate, and numerous secondary diseases. The first clinical signs may be observed as early as three days of age, although they are most commonly observed in 6- to 12-day-old chicks and may occur until 3 weeks of age. The chicks are frequently pale and may exhibit distention of the abdomen. In addition to smaller size and reduced body weight, other clinical signs were also observed in RSS chickens include poor feather development, listlessness, and diarrhea. The comb develops slowly, which is associated with impaired hearing and vision.

Why are RSS chickens easily observed in SLD chickens? Our study demonstrated the occurrence of mitochondrial abnormalities in the 7-week-old chicken's livers of RSS chickens and SLD chickens. RSS chicken's mitochondria dysfunction is more seriousness than SLD chickens, and we observed the abnormal nuclear genes related to mitochondrial function in 7-week-old RSS chickens and normal SLD chickens by expression patterns (Table 2), and found these genes involved in oxidative phosphorylation (OXPHOS) and other metabolic pathways.

The chicken mitochondrial genome is a $16.775-\mathrm{kb}$ circle of double-stranded DNA (Desjardins and Morais, 1990; Zavala, 2006) that encodes only 13 proteins, 2 rRNAs, and 22 tRNAs, which is very similar to the human mitochondrial genome (Desjardins and Morais, 1990). It has been reported that mutation or deletion of mitochondrial DNA (mtDNA) can induce mitochondrial disorders in humans (Zeviani et al., 1989; Moraes et al., 1991). As over 99\% of mitochondrial proteins are encoded by the nuclear genome, mitochondrial disorders can also be caused by mutations or/and abnormal expression of nuclear-encoded mitochondrial proteins (Bourgeron et al., 1995). In this study, we detected no mtDNA mutations in RSS chickens and SLD chickens.

Very little is currently known about mitochondrial diseases of chickens, and the etiology of RSS in chickens has plagued researchers for several decades. OXPHOS is the metabolic pathway in which cells use enzymes to oxidize nutrients, thereby releasing energy that is used to reform adenosine triphosphate (ATP). This pathway is a highly efficient way of generating energy, compared to alternative fermentation processes such as anaerobic glycolysis. OXPHOS also produces reactive oxygen species (ROS) which lead to the propagation of free radicals, damaging cells, and contributing to disease and possibly aging. The enzymes responsible for this metabolic pathway are also the targets of many drugs that inhibit their activities.

In this study, we demonstrated that mitochondrial membranes are destroyed in RSS chicken hepatocytes using transmission electron microscopy (TEM). Activity assays revealed that a series of complexes proteins (complex I, complex II, complex III, and complex IV) showed decreased enzyme activities in RSS chickens and SLD chickens, suggesting that the OXPHOS function was compromised.

\section{MATERIALS AND METHODS}

\section{Ethics Standards}

All of the animal experiments performed in this study were approved by South China Agriculture University's Institutional Animal Care and Use Committee (approval number SCAU\#0017), according to the regulations established by this committee and international standards for animal welfare.

\section{Animals}

The chickens were purchased from XinXing Poultry Breeding Company near Guangzhou, China. Three groups of chickens were used: RSS affected SLD chickens (RSS chickens) along with normal SLD chicken (SLD chickens) in 301 strain, and this strain is characterized by point mutations in exon 5 of the GHR gene as previously reported (Ouyang et al., 2012); normal chickens in 202 strain, which have a wild-type GHR gene. All of the chicks were provided with standard poultry feed and fresh water and reared at an optimum temperature $\left(27^{\circ} \mathrm{C}-37^{\circ} \mathrm{C}\right)$. After 7 weeks, six RSS chickens (three males and three females) and 6 SLD chickens ( 3 males and 3 females) from the 301 strain group, along with six normal chickens (three males and three females) from the 202 strain group, were collected. All of the experimental chicks were monitored periodically for signs of RSS, including body weight, abnormal feathering, uneven growth rate, poor performance, beak and leg color, lameness, and reluctance to move.

\section{Liver Histology}

Liver tissues were removed from each experimental chicken and fixed in formalin and $2.5 \%$ glutaraldehyde, respectively. The samples were stained using hematoxylin and eosin (H\&E) and Oil Red O, the slices were observed by optical microscopy, and photographed. We also used TEM (Hitachi HT-7700, Japan) to study the mitochondrial structure.

\section{Transmission Electron Microscopy}

Liver tissues were fixed in $2.5 \%$ glutaraldehyde for $4 \mathrm{~h}$ at $4^{\circ} \mathrm{C}$ and then cultured as previously described (Luo et al., 2016). Transmission electron microscope (Hitachi HT7700, Japan) was used to examine and photograph mitochondria, and five randomly selected areas were photographed at $\times 2500$ magnification and counted as previously reported $(\mathrm{Hu}$ et al., 2019).

\section{Quantitative Real-Time PCR}

To verify the data from microarray analysis (Table 2), quantitative real-time PCR (qRT-PCR) analysis was conducted to determine the relative expression levels of genes involved in the OXPHOS pathway in the livers of RSS chickens, SLD chickens, and normal chickens. Total RNA was extracted from the tissue samples using the RNAiso reagent (Takara, Japan) 
according to the manufacturer's instructions. The RNA integrity and concentration were determined using $1.5 \%$ agarose gel electrophoresis and a Nanodrop 2000c spectrophotometer (Thermo, USA), respectively. cDNA was carried out using a PrimeScript RT Reagent Kit (Takara) for RT-qPCR. The MonAmp ${ }^{\mathrm{TM}}$ ChemoHS qPCR Mix (Monad, China) was used for qRT-PCR in a Bio-Rad CFX96 Real-Time Detection instrument (Bio-Rad, USA) according to the manufacturer's protocol. Relative gene expression was measured by qRT-PCR twice for each reaction and nuclear gene $\beta$-actin was used as a control. The primers are listed in Table $\mathbf{1}$.

\section{Mitochondrial Protein Concentration}

The mitochondrial protein concentration was measured using a BCA protein assay kit (Beyotime, China) carried out in a Fluorescence/Multi-Detection Microplate Reader (BioTek, USA) according to the manufacturer's instructions.

\section{Enzyme Activities of Mitochondrial Respiratory Complexes}

Liver tissues were dissected, frozen in liquid nitrogen, and then stored at $-80^{\circ} \mathrm{C}$ (Thorburn et al., 2004). Enzyme activities of mitochondrial respiratory complexes were measured using commercial assay kits (Solarbio, China) according to the manufacturer's instructions carried out in a Fluorescence/ Multi-Detection Microplate Reader (BioTek, USA) according to the manufacturer's protocol. The enzyme activity of complex I was determined by the change in absorbance of NADH measured at $340 \mathrm{~nm}$. The enzyme activity of complex II was determined by the change in absorbance of 2, 6dichlorophenolindophenol measured at $600 \mathrm{~nm}$. The enzyme

TABLE 1 | Primers for qPCR analysis of nuclear gene expression and mtDNA content.

\begin{tabular}{|c|c|c|}
\hline Gene & Primer sequence $\left(5^{\prime}\right.$ to $\left.3^{\prime}\right)$ & Annealing temperature $\left({ }^{\circ} \mathrm{C}\right)$ \\
\hline F-NDUFB1 & TGTACAAGAGAGAGCTGAAGCC & 57.5 \\
\hline R-NDUFB1 & AGCAGTGACAAGTTGTAGGTGT & \\
\hline F-NDUFB2 & GCTGGGCCACTTCCCGTA & 64 \\
\hline R-NDUFB2 & AGTTCAAAACCCGCCCCTAC & \\
\hline F-NDUFB8 & CGATGCACTGGGACTTGAC & 65 \\
\hline R-NDUFB8 & TTGTAATGCGTCACAACCGG & \\
\hline F-NDUFB9 & GGCAGAAAGAGGTGAAGCAG & 65 \\
\hline R-NDUFB9 & CATACGTTCACTGGCACACC & \\
\hline F-NDUFB10 & CCAGCAAGCCAAGAACAAGT & 64.3 \\
\hline R-NDUFB10 & TTGGTAACCTGTGCAAGCT & \\
\hline F-NDUFB5 & TGAGACAGAGGGGAGATGGA & 64.3 \\
\hline R-NDUFB5 & CTCCACAGACAAGCCACAAT & \\
\hline F-NDUFB6 & GGTGTTCAACGCTTACCAGA & 64.3 \\
\hline R-NDUFB6 & CCGTCTCTAAAATTCTGTCCCC & \\
\hline F-IGF2BP3 & CAAGCTCTACATCGGCAACC & 65 \\
\hline R-IGF2BP3 & GGGACCGAATGCTCAACTTC & \\
\hline F-NDUFA8 & ACAAGGAGTTCATGCTGTGC & 57.5 \\
\hline R-NDUFA8 & CACCCAGCCCAACTTCTCTA & \\
\hline F-NDUFA9 & ATCCACTTCCACGTCCTCTC & 65 \\
\hline R-NDUFA9 & CTTCGCTGGTITGCTTCCT & \\
\hline F-SDHA & GGCACTGCTATGGTTACACG & 65 \\
\hline R-SDHA & ССССTTCCTTCCCGTATCTC & \\
\hline F-NDUFS4 & GGACTGGACTTCTCTGTGCT & 57 \\
\hline R-NDUFS4 & TGGCAGCAGGGAATACAGAA & \\
\hline
\end{tabular}

activities of complex III and complex IV were determined by the change in absorbance of reduced cytochrome $c$ measured at $550 \mathrm{~nm}$.

\section{Mitochondrial Respiratory Control Ratio}

The mitochondrial respiratory control ratio (RCR) was determined using an RCR assay kit (Genmed Scientifics Inc., USA) according to the manufacturer's instructions as previously described (Su et al., 2016). Oxygen consumption was measured using a Clarke-type oxygen electrode (Hansatech Oxytherm, UK). RCR was represented as the ratio of State III to State IV respiration rate. Data were normalized to the control group and expressed as percentage of control levels.

\section{ATP Concentration}

The ATP level was measured using an ATP assay kit (Beyotime, China) according to the manufacturer's instructions. A Fluorescence/Multi-Detection Microplate Reader (BioTek, USA) was used to determine the ATP level in gastrocnemius muscle and cells. Data were normalized to the control group and expressed as percentage of control levels.

\section{Mitochondrial Membrane Potential}

The mitochondrial membrane potential $(\Delta \Psi \mathrm{m})$ was measured using a JC-1 kit (Beyotime, China) according to the manufacturer's instructions. The fluorescence was determined using a Fluorescence/Multi-Detection Microplate Reader (BioTek, USA). Rotenone was used as standard inhibitor of $\Delta \Psi \mathrm{m}$. The $\Delta \Psi \mathrm{m}$ of mitochondria were represented as the ratio of JC-1 aggregated and JC-1 monomeric, and data were normalized to the control group and expressed as percentage of control levels.

\section{ROS Production}

The production of ROS was measured by the change in absorbance of formazan measured at $560 \mathrm{~nm}$ as previously described (Kim et al., 2003). Absorbance was determined using a Fluorescence/ Multi-Detection Microplate Reader (BioTek, USA).

\section{Statistical Analysis}

All the experiments were performed at least three times. The data were presented as means \pm standard error of the mean (S.E.M.), and the statistical analyses were performed using Student's $t$-test and the significance was represented by $\mathrm{P}$-values. $\mathrm{P}$ value $<0.05$ was considered to be statistically significant.

\section{RESULTS}

\section{RSS Chickens and SLD Chickens At the Same Age Exhibit Different Growth Stages} As shown in Figure 1, RSS chickens were smaller with villi faded slowly compared with SLD chickens. The cockscomb, legs, and beaks of the RSS chickens appeared pale in color, indicating a decline in blood production. Some of the chickens were found to have rickets, broken legs, or fractured toes. In addition, all RSS 


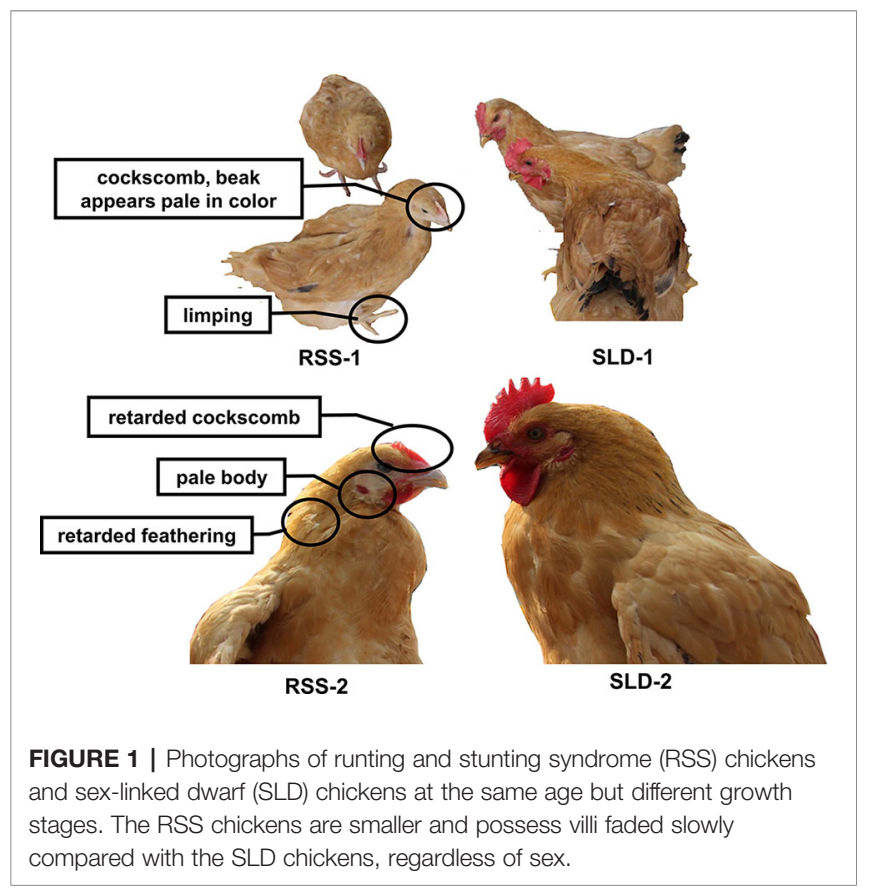

chickens were ALV-J and REV virus negative showed in Figure S1.

\section{Abnormally Expressed Genes in the Livers of RSS Chickens Compared With SLD Chickens Are Mainly in the OXPHOS Pathway, Fatty-Acid Metabolism Pathway, and Other Metabolic Pathways}

We next examined gene expression differences in the livers using expression profile chip analysis. Gene Set Enrichment Analysis results revealed that the RSS chickens exhibited abnormal expression of numerous genes involved in metabolic pathways (Table 2). We identified that many of the abnormally expressed genes were involved in OXPHOS pathway. These genes are associated with metabolic function in chickens. The results indicate a defective energy metabolism in RSS chickens compared with SLD chickens.

Complex I is the first complex in the electron transport chain (Hirst, 2005). The results revealed that the RSS chickens exhibited altered expression of the complex I genes. The change of the enzyme complex function directly or indirectly reflects the change of mitochondrial function. Furthermore, the IGF2BP3 and SDHA genes were abnormally expressed in RSS chicken livers.

\section{H\&E Staining and Oil Red O Staining of RSS Chickens, SLD Chickens and Normal Chickens}

Several viruses, bacteria, and other pathogens are believed to be responsible for RSS in broiler chickens. Bacteria are frequently isolated from RSS birds, which include Escherichia coli, Proteus mirabilis, Enterococcus faecium, Staphylococcus cohnii, Clostridium perfringens, Bacteroides fragilis, and Bacillus licheniformis. They are commonly found in the intestinal tract and may cause secondary infections that aggravate the initial lesions (Rebel et al., 2006). Some researchers have demonstrated that astrovirus may cause RSS in broilers (Kang et al., 2018). In this study, vacuolation of the hepatocytes was observed in both RSS and SLD chickens (Figures 2A-F). Hydrophic degeneration in the hepatocytes was also seen in the RSS chickens, which may be responsible for the liver cell vacuoles (Figures $\mathbf{2 A}, \mathbf{D}$ ). The hydrophic degeneration in the hepatocytes of RSS chickens may disrupt the structure and inhibited the function of mitochondria. This may further result in the destruction of the energy supply functions of the mitochondria.

\section{Mitochondrial Volume and Mitochondrial Structure of RSS Chickens, SLD Chickens, and Normal Chickens}

We next analyzed the structure of the mitochondria using TEM. The results revealed that the total volume and average volume of mitochondria which were reduced in RSS chickens compared with SLD chickens and normal chickens (Figures 3A, B). Furthermore, excessive amounts of vacuoles were present in the hepatocytes of RSS chickens. This may induce a decrease in the number of mitochondria. The inner and outer mitochondrial membranes exhibited different degrees of damage, and even disappeared, and the endoplasmic reticulum (ER) structure was also damaged (Figure 3C).

\section{Expression of Genes Involved in the OXPHOS Assessed by qRT-PCR}

To verify the consequences of expression profile chip analysis, the relative expression levels of genes involved in mitochondrial OXPHOS were determined in RSS chickens, SLD chickens, and normal chickens. These three groups of chickens were evaluated at 7 weeks of age to verify the microarray data. As shown in Figure 4, comparison of the female RSS chickens with SLD chickens and SLD chickens with normal chickens revealed that the IGF2BP3 and NDUFB2 genes were upregulated and the NDUFA8, NDUFA9, NDUFB1, NDUFB5, NDUFB6, NDUFB8,

TABLE 2 | Abnormally expressed genes in the livers of runting and stunting syndrome (RSS) chickens [relative to sex-linked dwarf (SLD) chickens].

Pathway Gene

Oxidative phosphorylation

Fatty-acid metabolism

Metabolism of

xenobiotics by

cytochrome P450
NDUFB5, NDUFB6, NDUFB8, NDUFB9, ATP6V1G1, NDUFB1, NDUFB2, ATP6VOC, NDUFS4, ATP5I, TCIRG1, NDUFB10, NDUFA8, NDUFA9, NDUFA7, ATP5F1, ATP6V1D, LOC770190, PPA1, COX6C, SDHA, ATP6V1E1, COX6A1, ATP6VOA2, LOC770879 ALDH7A1, CPT2, ADH5, ACADL, ACAT2, ALDH3A2, ACSL5 CYP3A37, ADH5, EPHX1, LOC421447, LOC396380, UGT1A1, CYP3A80 
A

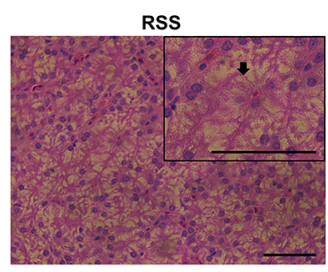

D

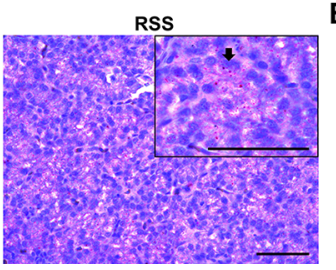

B

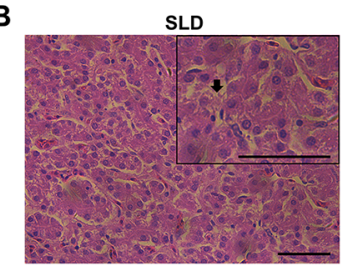

E

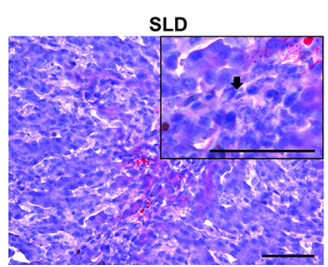

C

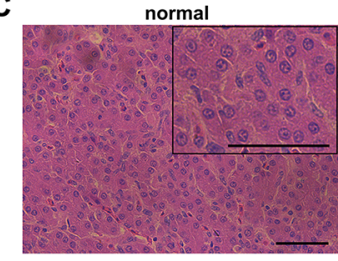

F

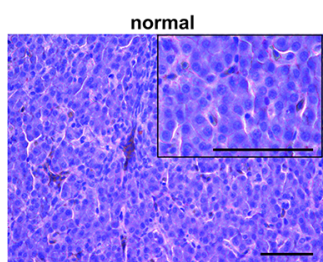

FIGURE 2 | Liver tissues stained with H\&E and Oil Red O. (A) Liver section stained with H\&E from runting and stunting syndrome (RSS) chicken. Bar, 50 um. As indicated by the arrow, the hepatocytes possess a sparse cytoplasm and contain a large number of vacuoles. The hepatocytes are not only abnormal in terms of morphology and structure but also present in reduced number. (B) Liver section stained with H\&E from sex-linked dwarf (SLD) chicken. Bar, 50 mm. Compared with the hepatocytes from the RSS chicken, the hepatocytes of the normal SLD chicken contain fewer vacuoles and less sparse cytoplasm. However, compared with the hepatocytes from the normal chicken, the hepatocytes of the SLD chicken possess a sparse cytoplasm and contain a larger number of vacuoles. (C) Liver section stained with H\&E from normal chicken. Bar, $50 \mu \mathrm{m}$. The hepatocytes possess a normal shape and structure, and no vacuoles and sparse cytoplasm are observed. (D, E) Liver sections stained with Oil red O from RSS and SLD chickens, respectively, showing vacuolar hydropic degeneration, which leads to an incomplete structure of hepatocytes. Bar, $50 \mu \mathrm{m}$. These phenomena were more severe observed in the RSS chickens. (F) Liver section stained with Oil red O from normal chicken. Bar, $50 \mu \mathrm{m}$. No abnormal vacuoles in the hepatocytes is evident.

NDUFB9, NDUFB10, NDUFS4, and SDHA genes were downregulated. Moreover, some differences were also observed in the male RSS chickens. In particular, the NDUFA8, NDUFA9, NDUFB1, NDUFB5, NDUFB6, NDUFB8, NDUFB9, NDUFB10, NDUFS4, and SDHA genes were upregulated and the NDUFB2 genes were downregulated in the livers of male RSS chickens, which is contrary to the consequences of female RSS chickens except for IGF2BP3 gene (Figure 5). These results suggest that the IGF2BP3 and SDHA genes, as major genes related to growth and energy supply, are associated with the development of RSS in chickens.

\section{Mitochondrial Function Measurement}

The activities of mitochondrial enzymes in the liver, including OXPHOS complexes, are summarized in Figure 6. The enzyme activities of complex I, complex II, complex III, and complex IV in the RSS chickens were reduced by $45 \%, 24 \%, 21 \%$, and $41 \%$, respectively, compared with SLD chickens (Figures 6A-D). Similarly, the enzyme activities of complex I, complex II, complex III, and complex IV in the SLD chickens were reduced by $56 \%, 22 \%, 21 \%$, and $61 \%$, respectively, compared with normal chickens. To determine whether these changes in the activities of the respiratory complexes influenced the function of the mitochondria, we next assessed the mitochondrial function by measuring the RCR, ATP level, $\Delta \Psi \mathrm{m}$, and ROS production. The RCR values of the RSS chickens was reduced by $15 \%$ compared with SLD chickens, and that of SLD chickens was reduced by 35\% compared with normal chickens (Figure 6E), indicating a lower oxygen consumption in the RSS chickens and SLD chickens. The ATP level of the RSS chickens was reduced by $37 \%$ compared with
SLD chickens, and that of SLD chickens was reduced by $25 \%$ compared with normal chickens (Figure 6F), indicating a lower efficiency of ATP production in the RSS chickens and SLD chickens. The $\Delta \Psi \mathrm{m}$ measured by JC- 1 of the RSS chickens was reduced by $16 \%$ compared with SLD chickens, and that of SLD chickens was reduced by $21 \%$ compared with normal chickens (Figure 6G), indicating a dissipative $\Delta \Psi \mathrm{m}$ in the RSS chickens and SLD chickens. Finally, the production of ROS in the RSS chickens was increased by $14 \%$ compared with SLD chickens, and that of SLD chickens was increased by $22 \%$ compared with normal chickens (Figure 6H).

\section{DISCUSSION}

The signs of RSS are usually observed in meat-type chickens between 3 and 6 weeks of age. Previous work has demonstrated that certain strains of birds appear to be more susceptible to the effects of RSS than others, and that male birds are more severely affected than females (Zavala, 2006). However, it is interesting to note that RSS-resistant broiler strains have stronger immunological responses against bacterial and viral infections than RSS-susceptible strains (Rebel et al., 2006). Some researchers have suggested that the poor growth and retarded feathering consistently observed in RSS-affected birds are due to a chronic infection. However, we found that SLD chickens were more susceptible to the effects of RSS and exhibited weaker immunological responses than normal chickens. Therefore, we studied 7-week-old yellow-feather broilers with RSS like features. Of note, No indication of viral was also detected in the liver 
A

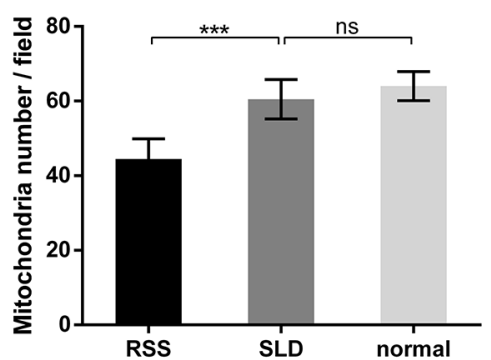

C

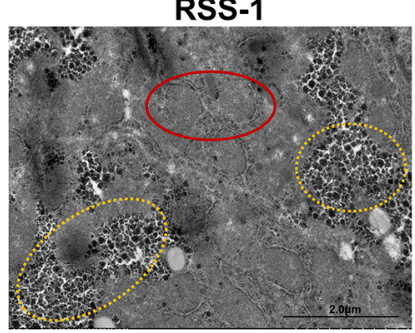

SLD-1

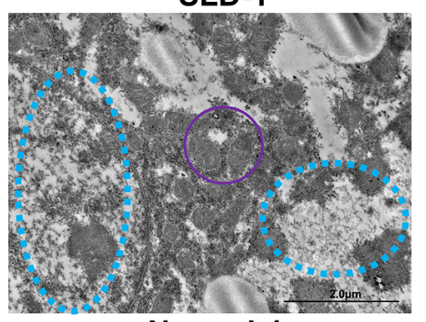

Normal-1

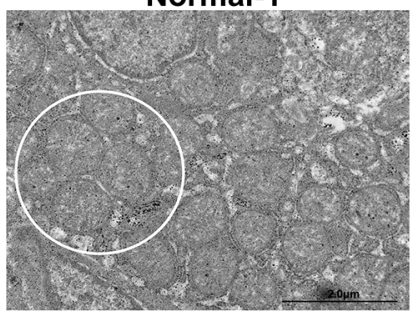

B

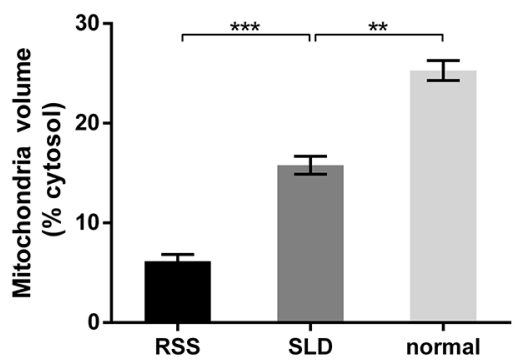

RSS-2

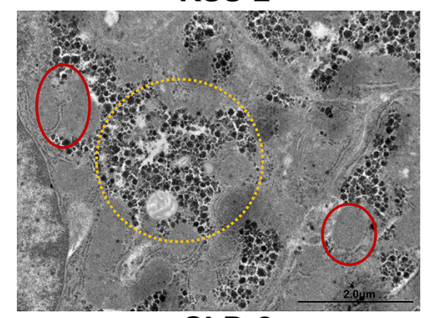

SLD-2

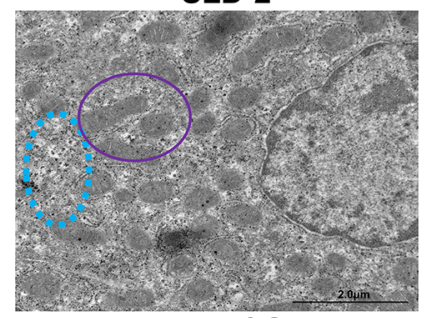

normal-2

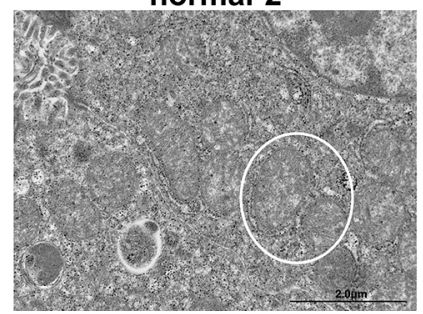

RSS-3

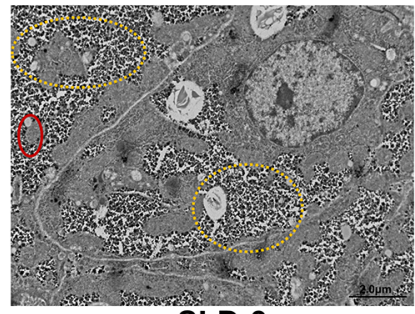

SLD-3

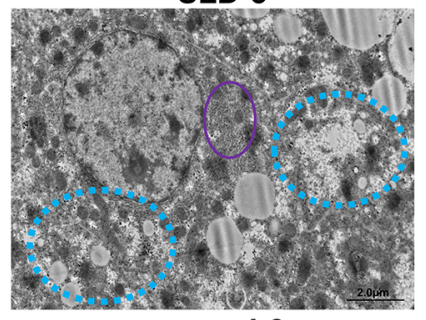

normal-3

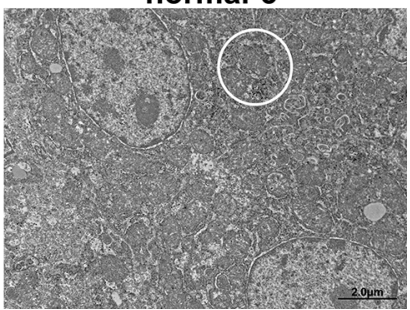

FIGURE 3 | Total and average volumes of mitochondria and transmission electron microscopy (TEM) images. Total volume (A) and average volume of mitochondria (B) in runting and stunting syndrome (RSS) chickens, sex-linked dwarf (SLD) chickens, and normal chickens. To examine the mitochondrial morphology, five randomly selected areas were imaged at 25,000x magnification and analyzed. (C) RSS 1-3: TEM images of liver sections from 7-week-old RSS chickens. In this group, vacuoles in the hepatocytes had destroyed the mitochondria and other organelles, as indicated by the yellow circles. This not only caused a severe decrease in the number of mitochondria, leading to the destruction of the interactions between mitochondria and other organelles, but also seriously affected the structure and function of the mitochondria. As indicated by the red circles, the mitochondria were small in size and their inner and outer membranes had been destroyed or even disappeared. The mitochondrial cristae had also disappeared. SLD 1-3: TEM images of liver sections from 7-week-old SLD chickens. In this group, the mitochondria in the hepatocytes are surrounded by a large number of vacuoles, and the number of mitochondria is also reduced. The mitochondria are small in size and their structure is incomplete, although the degree of damage is less than that in the RSS chickens. Vacuoles and parts of destroyed mitochondria are indicated by blue and purple circles, respectively. Normal 1-3: TEM images of liver sections from 7-week-old normal chickens. In this group, normal mitochondria (indicated by white circles) and other organelles are observed. They are not only complete in structure but also present in large numbers. Data are represented as the mean \pm SEM; ${ }^{* *} p$ $<0.01 ;{ }^{* \star *} p<0.001 ; \mathrm{ns}$, no significan difference.

tissues of the RSS chickens by viruses detection as previously described (Zavala, 2006; Zhang et al., 2015).

Further, we revealed that the mRNA of the insulin-like growth factor 2 mRNA-binding protein 3 (IGF2BP3) was upregulated in the RSS chicken's liver tissue. IGF2BP3 plays an important role in the formation and growth of many tumors and is typically expressed in embryonic tissue, muscles, and the placenta. In fact, the expression of IGF2BP3 is low in the liver tissues of normal chickens. IGF2BP3 is an oncofetal protein with high expression during embryogenesis, low expression in adult tissues, and re-expression in malignant tissues (Palanichamy et al., 2016a). Another study revealed that IGF2BP3, as a 


\section{Female}
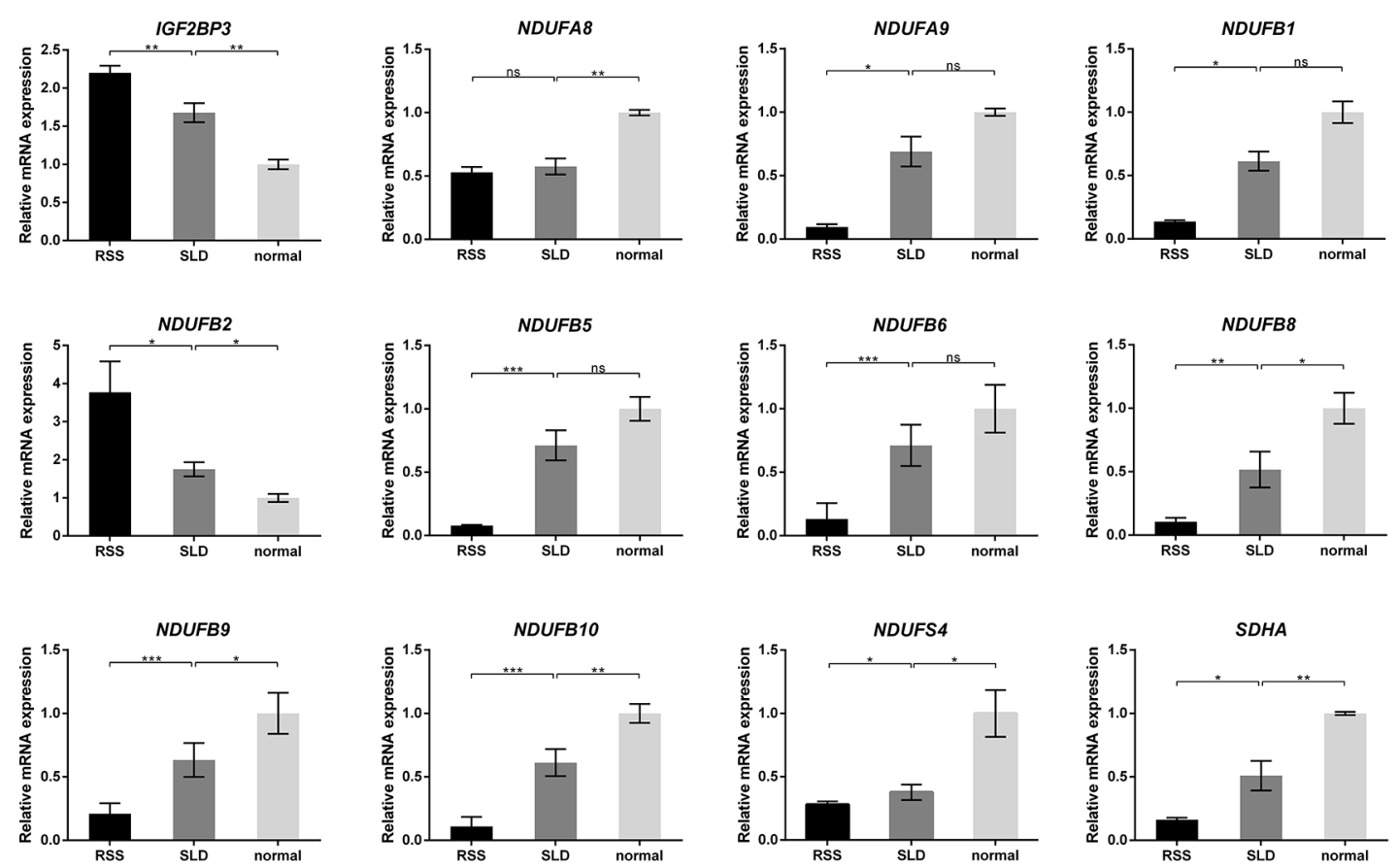

FIGURE 4 | Relative expression of IGF2BP3 and other genes involved in oxidative phosphorylation (OXPHOS) in the livers of female chickens. Comparison of the female runting and stunting syndrome (RSS) chickens with sex-linked dwarf (SLD) chickens and SLD chickens with normal chickens revealed that the IGF2BP3 and NDUFB2 genes were upregulated and the NDUFA8, NDUFA9, NDUFB1, NDUFB5, NDUFB6, NDUFB8, NDUFB9, NDUFB10, NDUFS4, and SDHA genes were downregulated. Data are represented as the mean $\pm \mathrm{SEM} ;{ }^{\star} p<0.01 ;{ }^{\star \star} p<0.01 ;{ }^{\star \star \star} p<0.001$; ns, no significant difference.

human ontogenic master switch, restricts megakaryocyte development by modulating a lineage-specific $\mathrm{P}$-TEFb activation mechanism, revealing potential strategies toward enhancing platelet production (Elagib et al., 2017). In this study, the expression of IGF2BP3 in RSS chickens was examined by qRT-PCR. We found that IGF2BP3 expression was increased in the livers of RSS chickens and SLD chickens. This raises the question of the nature of the association between enhanced IGF2BP3 expression and RSS in chickens. Minchenko et al. demonstrated that the IGF2BP3 gene is expressed in hypoxic tissues, indicating its participation in the regulation of metabolic and proliferative processes via IGF/INS receptors (Minchenko et al., 2015). In this study, we found that both the livers of RSS and SLD chickens exhibited signs of mitochondrial dysfunction, and the livers exhibited black or yellow in color. This suggests that the livers of RSS chickens are hypoxic. This would explain why IGF2BP3 expression was increased in the livers of the RSS chickens. The RCR results further demonstrated that the livers of RSS chickens were hypoxic compared with SLD chickens. The abnormal expression of IGF2BP3 confirmed that oxidative respiratory dysfunction occurs in the mitochondria of both RSS chickens and SLD chickens. Under hypoxic conditions, $\mathrm{NADH}$ and $\mathrm{FADH}_{2}$ cannot participate in respiratory chain regeneration, thereby interrupting the OXPHOS and preventing the normal synthesis of ATP. Our results also demonstrated that the ATP production was reduced in the livers of RSS chickens.

Moreover, we also revealed that the amounts of several components of the OXPHOS pathway in RSS chickens. NDUFA8, NDUFA9, NDUFB1, NDUFB5, NDUFB6, NDUFB8, NDUFB9, NDUFB10, and NDUFS4 are downregulated in female RSS chickens compared to SLD chickens. However, the expression of these genes of female RSS chickens is contrary to that of male RSS chickens, which may indicate that the regulatory mechanisms of male and female RSS chickens are different. In particular, succinate dehydrogenase complex subunit A (SDHA) was abnormal expression in the livers of RSS chickens compared with those of SLD chickens. SDHA is anchored to the inner mitochondrial membrane by one hydrophobic subunit of SDHC (Sun et al., 2005). The catalytic subunit of SDHA is hydrophilic and extends into the mitochondrial matrix (Lancaster and Simon, 2002). SDHA contains a covalently attached flavin adenine dinucleotide (FAD) cofactor and the succinate-binding site. Flavination is important for succinate oxidation (Robinson et al., 1994). A previous study confirmed that flavination defects interfere with 


\section{Male}
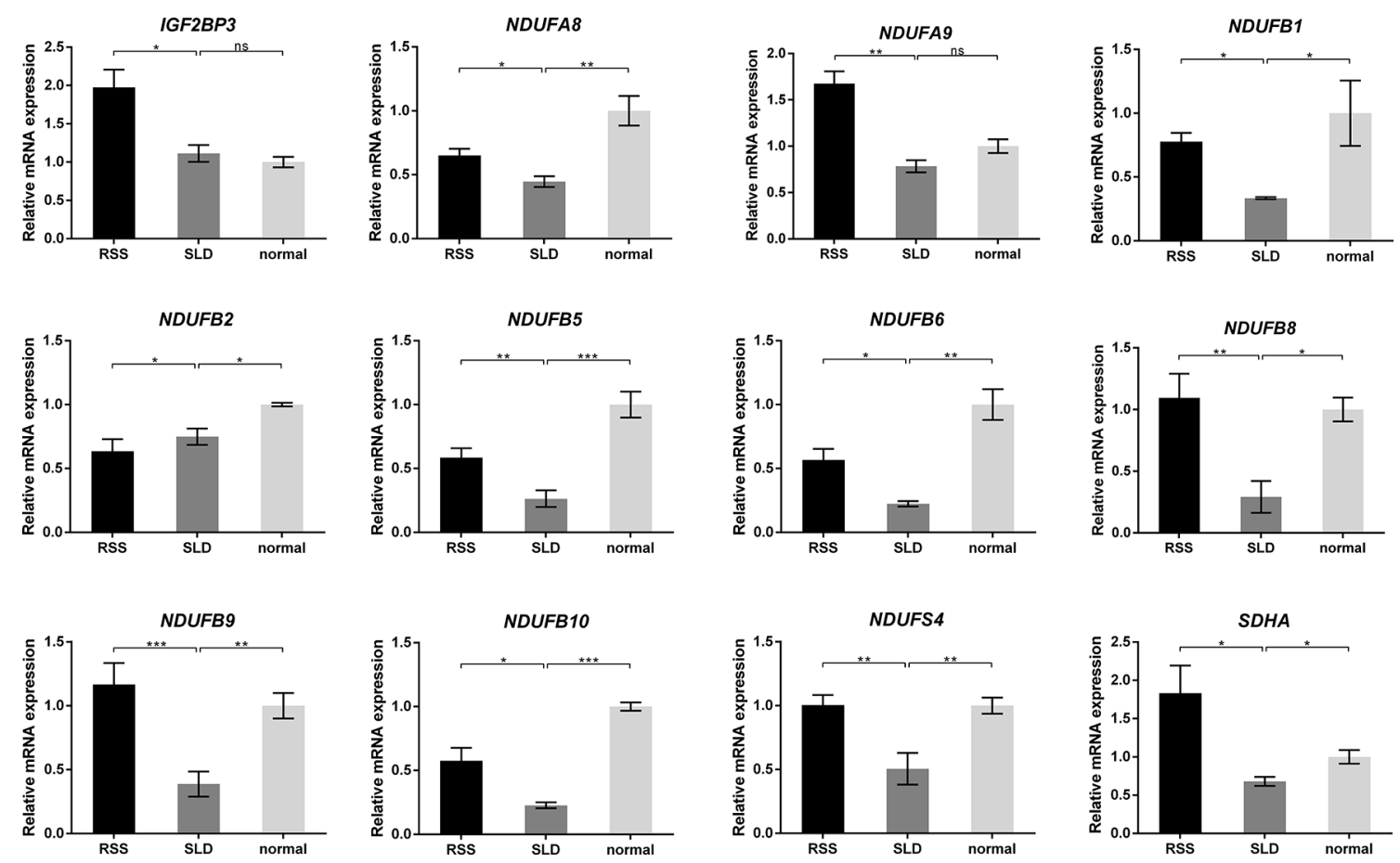

FIGURE 5 | Relative expression of IGF2BP3 and other genes involved in oxidative phosphorylation (OXPHOS) in the livers of male chickens. Comparison of the male RSS chickens with SLD chickens the IGF2BP3, NDUFA8, NDUFA9, NDUFB1, NDUFB5, NDUFB6, NDUFB8, NDUFB9, NDUFB10, NDUFS4, and SDHA genes were upregulated and the NDUFB2 genes were downregulated. Comparison of the male SLD chickens with normal chickens the /GF2BP3 were upregulated and the NDUFA8, NDUFA9, NDUFB1, NDUFB2, NDUFB5, NDUFB6, NDUFB8, NDUFB9, NDUFB10, NDUFS4, and SDHA genes were downregulated. Data are represented as the mean \pm SEM; ${ }^{\star} p<0.01 ;{ }^{\star \star} p<0.01 ;{ }^{\star \star \star} p<0.001$.

SDHA activity, likely reducing the overall growth rates in vitro and in vivo that have been observed in experimental models of SDHA deficiency (Guzy et al., 2008). Another study demonstrated that knockdown of the SDHA/B genes in HeLa cells led to the accumulation of succinate (Xiao et al., 2012). Konieczna et al. found that partial silencing of SDHA with specific siRNAs resulted in either less efficient or delayed entrance of the cells to the S phase (Konieczna et al., 2015). However, the cells remained viable. The results of this study revealed that SDHA was abnormal expression in the livers of RSS chickens compared with SLD chickens, but no mutation was detected. Lower OXPHOS activity will lead to insufficient energy supply, which appears to be one of the reasons for the development of RSS in chickens.

Decreased OXPHOS activities, along with higher ROS production in RSS and SLD chickens, are indicative of damaged mitochondrial function and efficiency. The vacuolar hydropic degeneration may induce the enlargement of hepatocytes and compress adjacent organelles, leading to damage of the nuclei and mitochondria of liver cells. This phenomenon was observed not only in the RSS chickens but also in SLD chickens with GHR mutation. Previous studies have confirmed that growth hormone $(\mathrm{GH})$ stimulates cellular oxygen consumption in $\mathrm{CHO}$ cells transfected with cDNA coding for the full-length GHR (Perret-Vivancos et al., 2006a). GH affects mitochondrial metabolism by indirectly regulating the activities of OXPHOS enzyme complexes (Brown-Borg et al., 2012). We found that the abnormal interaction between GH and GHR can influence mitochondrial oxidation and respiratory functions. The five multimeric enzyme complexes that drive OXPHOS and cellular respiration are contained within the inner mitochondrial membrane (Barrientos et al., 2009). Determining the activities of individual enzymes in the respiratory chain complex provides an important diagnostic indicator of mitochondrial disease (Frazier and Thorburn, 2012). In mitochondrial disease, the activities of single or multiple complexes are reduced in the affected tissue (ElHattab and Scaglia, 2013). Activity assays typically reveal decreased activities of complex I, complex III, and complex IV in liver tissues (El-Hattab and Scaglia, 2013). In this study, we observed decreased enzyme activities of complex I, complex II, complex III, and complex IV in RSS and SLD chickens.

Inefficient mitochondrial function was also observed upon measuring the RCR, ATP level, $\Delta \Psi \mathrm{m}$, and ROS production. As mitochondria are responsible for the majority of oxygen consumption, the RCR is an essential parameter for studying 
A

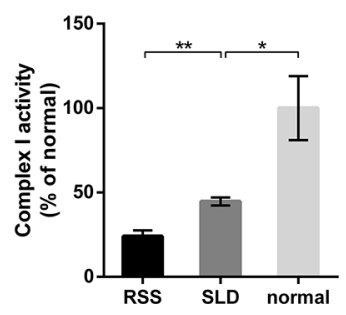

E

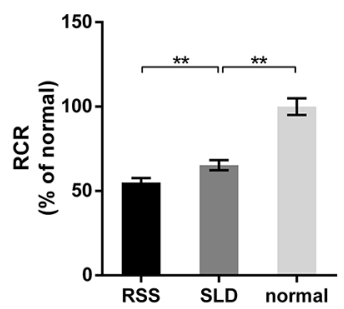

B

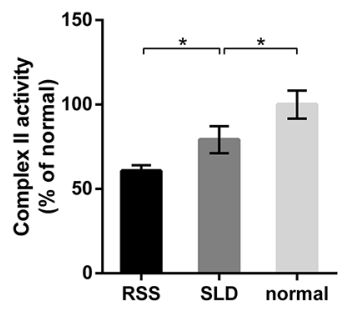

F

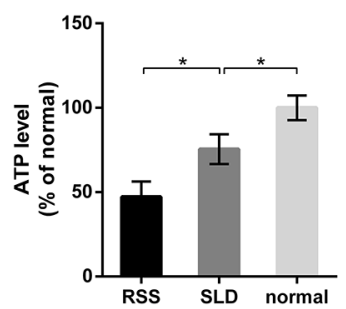

C

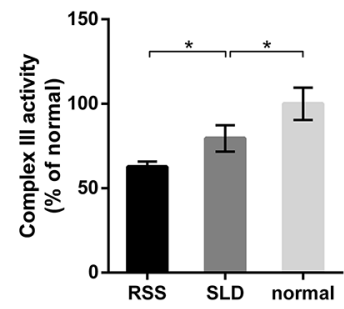

G

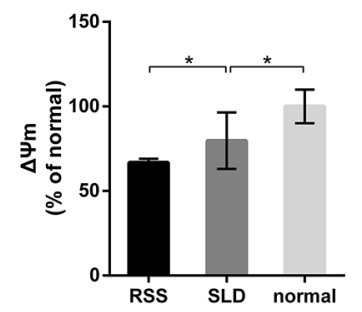

D

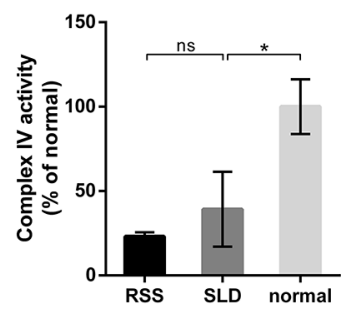

H

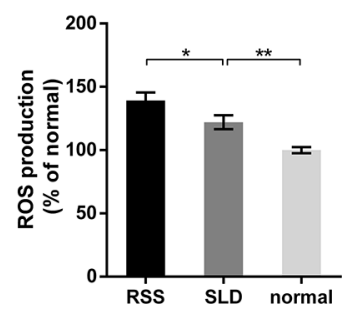

FIGURE 6 | Enzymatic activities of mitochondrial respiratory complexes and mitochondrial function analysis of 7-week-old runting and stunting syndrome (RSS) (RSS) chickens, sex-linked dwarf (SLD) chickens, and normal chickens. The enzymatic activities of (A) complex I, (B) complex II, (C) complex III, and (D) complex IV in the livers of RSS chickens, SLD chickens, and normal chickens expressed a percentage of normal chickens. Respiratory control ratio (RCR) (E), adenosine triphosphate (ATP) level (F), $\Delta \Psi m(\mathbf{G})$, and reactive oxygen species (ROS) production (H) in the livers of RSS, SLD, and normal chickens expressed as a percentage of normal chickens. Data are represented as the mean $\pm \mathrm{SEM} ;{ }^{*} p<0.01 ;{ }^{* *} p<0.01$; ns, no significant diffrerence.

mitochondrial function (Frezza et al., 2007). Meanwhile, $\Delta \Psi \mathrm{m}$ is essential for mitochondrial function, and mitochondrial dysfunction is normally accompanied by a decrease in $\Delta \Psi \mathrm{m}$ (Javadov et al., 2006). Further, excessive ROS production can impair the activities of mitochondrial respiratory complexes and reduced ATP production (Sudheesh et al., 2013). Here, we observed alterations in the RCR, ATP level, $\Delta \Psi \mathrm{m}$, and ROS production in both RSS chickens and SLD chickens. However, the mitochondrial function was lowest in RSS chickens, indicating further mitochondrial dysfunction in RSS chickens compared with SLD chickens. This may further impair immunity, causing greater mortality in RSS chickens.

Mitochondria, the only organelles in animal cells that contain their own genome, exchange $\mathrm{Ca}^{2+}$ and ROS with the endoplasmic reticulum (Pacher and Hajnoczky, 2001; Zorov et al., 2014; Booth et al., 2016). An evolutionarily conserved machinery enables the complete and sequential fusion of the outer and inner mitochondrial membranes (Robinson et al., 1994). In this study, no inflammation was detected by $H \& E$ staining and no lymphocyte infiltration was observed. Vacuolation of hepatocytes was observed, and the cytoplasm was disappeared in RSS and SLD chickens. The hydropic degeneration in the hepatocytes was the main cause of the liver cell vacuoles. GHR mutation induces an abnormal interaction between GH and GHR. As GHR gene is associated with oxidative respiration (Perret-Vivancos et al., 2006b), this may further influences mitochondrial oxidation and respiratory functions. Here, the TEM results revealed that the inner and outer mitochondrial membranes of the RSS chickens exhibited varying degrees of damage or had even disappeared. Consequently, the five multimeric enzyme complexes within the inner mitochondrial membrane are unable to drive OXPHOS and cellular respiration. The GHR mutation, along with contributions from the additional factors mentioned above, may lead to the hindered growth and development in SLD chickens. If mitochondrial damage becomes more severe in SLD chickens, these may exhibit the signs of RSS. The methods reported in this study can also be used as a model for inner mitochondrial membrane GHR mutation.

Together, all of these changes in mitochondrial functions could account for mitochondria dysfunction, resulting in poor weight gain and retarded growth or stunting of chicks. Our findings revealed that the RSS in chicken is caused by mitochondria dysfunction. We didn't find mtDNA mutation in RSS and SLD chickens. The GHR mutation may cause mitochondria dysfunction in SLD chicken which is induced by GHR mutation, but RSS chicken's mitochondria dysfunction is more serious. We hypothesize that RSS chicken's mitochondrial disorders are caused by other nuclear gene mutations.

\section{ETHICS STATEMENT}

All procedures involving animals were approved by South China Agriculture University's Institutional Animal Care and Use Committee (approval number SCAU\#0017), according to the 
regulations established by this committee. Animals involved in this study were humanely sacrificed as necessary.

\section{AUTHOR CONTRIBUTIONS}

$\mathrm{HL}$ and $\mathrm{BH}$ contributed equally to this manuscript. HL designed the study, and wrote the paper. $\mathrm{BH}$ carried out experiments, and analyzed data. QL and SH participated in the design of the experiment and data analysis. YLu, BZ, and YG participated in data collection and interpretation, and helped with performing some of the manuscripts' experiments. YLi, MS, and QN helped for useful discussion and language correction. $\mathrm{XZ}$ and $\mathrm{DZ}$ participated in the design, manuscript writing and final approval of the manuscript. All authors read and approved the final manuscript.

\section{REFERENCES}

Barrientos, A., Fontanesi, F., and Diaz, F. (2009). Evaluation of the mitochondrial respiratory chain and oxidative phosphorylation system using polarography and spectrophotometric enzyme assays. Curr. Protoc. Hum. Genet. 19, 4. doi: 10.1002/0471142905.hg1903s63

Booth, D. M., Enyedi, B., Geiszt, M., Varnai, P., and Hajnoczky, G. (2016). Redox nanodomains are induced by and control calcium signaling at the ERmitochondrial interface. Mol. Cell 63, 240-248. doi: 10.1016/j.molcel.2016.05.040

Bourgeron, T., Rustin, P., Chretien, D., Birch-Machin, M., Bourgeois, M., ViegasPequignot, E., et al. (1995). Mutation of a nuclear succinate dehydrogenase gene results in mitochondrial respiratory chain deficiency. Nat. Genet. 11, 144149. doi: $10.1038 / \mathrm{ng} 1095-144$

Bracewell, C. D., and Wyeth, P. (1981). Infectious stunting of chickens. Vet. Rec. 109, 64. doi: 10.1136/vr.109.3.64-b

Brown-Borg, H. M., Johnson, W. T., and Rakoczy, S. G. (2012). Expression of oxidative phosphorylation components in mitochondria of long-living Ames dwarf mice. Age (Dordr) 34, 43-57. doi: 10.1007/s11357-011-9212-x

Desjardins, P., and Morais, R. (1990). Sequence and gene organization of the chicken mitochondrial genome. A novel gene order in higher vertebrates. J. Mol. Biol. 212, 599-634. doi: 10.1016/0022-2836(90)90225-B

Elagib, K. E., Lu, C. H., Mosoyan, G., Khalil, S., Zasadzinska, E., Foltz, D. R., et al. (2017). Neonatal expression of RNA-binding protein IGF2BP3 regulates the human fetal-adult megakaryocyte transition. J. Clin. Invest. 127, 2365-2377. doi: $10.1172 /$ JCI88936

El-Hattab, A. W., and Scaglia, F. (2013). Mitochondrial DNA depletion syndromes: review and updates of genetic basis, manifestations, and therapeutic options. Neurotherapeutics 10, 186-198. doi: 10.1007/s13311-013-0177-6

Frazier, A. E., and Thorburn, D. R. (2012). Biochemical analyses of the electron transport chain complexes by spectrophotometry. Methods Mol. Biol. 837, 4962. doi: 10.1007/978-1-61779-504-6_4

Frezza, C., Cipolat, S., and Scorrano, L. (2007). Organelle isolation: functional mitochondria from mouse liver, muscle and cultured fibroblasts. Nat. Protoc. 2, 287-295. doi: 10.1038/nprot.2006.478

Guzy, R. D., Sharma, B., Bell, E., Chandel, N. S., and Schumacker, P. T. (2008). Loss of the SdhB, but Not the SdhA, subunit of complex II triggers reactive oxygen species-dependent hypoxia-inducible factor activation and tumorigenesis. Mol. Cell. Biol. 28, 718-731. doi: 10.1128/mcb.01338-07

Hirst, J. (2005). Energy transduction by respiratory complex I-an evaluation of current knowledge. Biochem. Soc. Trans. 33, 525-529. doi: 10.1042/ BST0330525

Hu, B., Hu, S., Yang, M., Liao, Z., Zhang, D., Luo, Q., et al. (2019). Growth hormone receptor gene is essential for chicken mitochondrial function in vivo and in vitro. Int. J. Mol. Sci. 20, 7. doi: 10.3390/ijms20071608

Javadov, S., Baetz, D., Rajapurohitam, V., Zeidan, A., Kirshenbaum, L. A., and Karmazyn, M. (2006). Antihypertrophic effect of $\mathrm{Na}+\mathrm{H}+$ exchanger isoform 1 inhibition is mediated by reduced mitogen-activated protein kinase activation secondary to improved mitochondrial integrity and decreased generation of

\section{FUNDING}

This work was supported by grants from the National Natural Science Fund Committee National Youth Project (Project grant no: 31401046), Guangdong Provincial Promotion Project on Preservation and Utilization of Local Breed of Livestock and Poultry and Guangdong Youth Talent Project.

\section{SUPPLEMENTARY MATERIAL}

The Supplementary Material for this article can be found online at: https://www.frontiersin.org/articles/10.3389/fgene.2019. 01337/full\#supplementary-material

mitochondrial-derived reactive oxygen species. J. Pharmacol. Exp. Ther. 317, 1036-1043. doi: 10.1124/jpet.105.100107

Kang, K. I., Linnemann, E., Icard, A. H., Durairaj, V., Mundt, E., and Sellers, H. S. (2018). Chicken astrovirus as an aetiological agent of runting-stunting syndrome in broiler chickens. J. Gen. Virol. 99, 512-524. doi: 10.1099/ jgv.0.001025

Kim, T. S., Yun, B. Y., and Kim, I. Y. (2003). Induction of the mitochondrial permeability transition by selenium compounds mediated by oxidation of the protein thiol groups and generation of the superoxide. Biochem. Pharmacol. 66, 2301-2311. doi: 10.1016/j.bcp.2003.08.021

Konieczna, A., Szczepanska, A., Sawiuk, K., Wegrzyn, G., and Lyzen, R. (2015). Effects of partial silencing of genes coding for enzymes involved in glycolysis and tricarboxylic acid cycle on the enterance of human fibroblasts to the $S$ phase. BMC Cell Biol. 16, 16. doi: 10.1186/s12860-015-0062-8

Kouwenhoven, B., Davelaar, F. G., and Van Walsum, J. (1978). Infectious proventriculitis causing runting in broilers. Avian Pathol. 7, 183-187. doi: $10.1080 / 03079457808418269$

Lancaster, C. R., and Simon, J. (2002). Succinate:quinone oxidoreductases from epsilon-proteobacteria. Biochim. Biophys. Acta 1553, 84-101. doi: 10.1016/ S0005-2728(01)00230-4

Luo, W., Lin, S., Li, G., Nie, Q., and Zhang, X. (2016). Integrative analyses of miRNA-mRNA interactions reveal let-7b, miR-128 and MAPK pathway involvement in muscle mass loss in sex-linked dwarf chickens. Int. J. Mol. Sci. 17, 276. doi: 10.3390/ijms17030276

Meldau, S., De Lacy, R. J., Riordan, G., Goddard, E. A., Pillay, K., Fieggen, K. J., et al. (2018). Identification of a single MPV17 nonsense-associated altered splice variant in 24 South African infants with mitochondrial neurohepatopathy. Clin. Genet. 93, 1093-1096. doi: 10.1111/cge.13208

Minchenko, D. O., Kharkova, A. P., Karbovskyi, L. L., and Minchenko, O. H. (2015). Expression of insulin-like growth factor binding protein genes and its hypoxic regulation in U87 glioma cells depends on ERN1 mediated signaling pathway of endoplasmic reticulum stress. Endocr. Regul. 49, 73-83. doi: 10.4149/endo_2015_04_185

Moraes, C. T., Shanske, S., Tritschler, H. J., Aprille, J. R., Andreetta, F., Bonilla, E., et al. (1991). mtDNA depletion with variable tissue expression: a novel genetic abnormality in mitochondrial diseases. Am. J. Hum. Genet. 48, 492-501. doi: 10.1016/0378-1119(91)90142-X

Ouyang, J., Xie, L., Nie, Q., Zeng, H., Peng, Z., Zhang, D., et al. (2012). The effects of different sex-linked dwarf variations on chinese native chickens. J. Integr. Agr. 11, 1500-1508.

Pacher, P., and Hajnoczky, G. (2001). Propagation of the apoptotic signal by mitochondrial waves. EMBO J. 20, 4107-4121. doi: 10.1093/emboj/20.15.4107

Palanichamy, J. K., Tran, T. M., Howard, J. M., Contreras, J. R., Fernando, T. R., Sterne-Weiler, T., et al. (2016a). RNA-binding protein IGF2BP3 targeting of oncogenic transcripts promotes hematopoietic progenitor proliferation. J. Clin. Invest. 126, 1495-1511. doi: 10.1172/JCI80046

Perret-Vivancos, C., Abbate, A., Ardail, D., Raccurt, M., Usson, Y., Lobie, P. E., et al. (2006a). Growth hormone activity in mitochondria depends on $\mathrm{GH}$ 
receptor Box 1 and involves caveolar pathway targeting. Exp. Cell Res. 312, 215-232. doi: 10.1016/j.yexcr.2005.10.027

Perret-Vivancos, C., Abbate, A., Ardail, D., Raccurt, M., Usson, Y., Lobie, P. E., et al. (2006b). Growth hormone activity in mitochondria depends on GH receptor Box 1 and involves caveolar pathway targeting. Exp. Cell Res. 312, 215-232. doi: 10.1016/j.yexcr.2005.10.027

Rebel, J. M. J., Balk, F. R. M., Post, J., Hemert, S. V., and Stockhofe, B. Z. N. (2006). Malabsorption syndrome in broilers. World. Poultry Sci. J. 62, 17-30. doi: 10.1079/WPS200481

Robinson, K. M., Rothery, R. A., Weiner, J. H., and Lemire, B. D. (1994). The covalent attachment of FAD to the flavoprotein of Saccharomyces cerevisiae succinate dehydrogenase is not necessary for import and assembly into mitochondria. Eur. J. Biochem. 222, 983-990. doi: 10.1111/j.1432-1033.1994.tb18949.x

Sarzi, E., Goffart, S., Serre, V., Chretien, D., Slama, A., Munnich, A., et al. (2007). Twinkle helicase (PEO1) gene mutation causes mitochondrial DNA depletion. Ann. Neurol. 62, 579-587. doi: 10.1002/ana.21207

Su, Y., Zhu, L., Yu, X., Cai, L., Lu, Y., Zhang, J., et al. (2016). Mitochondrial transplantation attenuates airway hyperresponsiveness by inhibition of cholinergic hyperactivity. Theranostics 6, 1244-1260. doi: 10.7150/thno.13804

Sudheesh, N. P., Ajith, T. A., and Janardhanan, K. K. (2013). Ganoderma lucidum ameliorate mitochondrial damage in isoproterenol-induced myocardial infarction in rats by enhancing the activities of TCA cycle enzymes and respiratory chain complexes. Int. J. Cardiol. 165, 117-125. doi: 10.1016/j.ijcard.2011.07.103

Sun, F., Huo, X., Zhai, Y., Wang, A., Xu, J., Su, D., et al. (2005). Crystal structure of mitochondrial respiratory membrane protein complex II. Cell 121, 1043-1057. doi: 10.1016/j.cell.2005.05.025

Thorburn, D. R., Chow, C. W., and Kirby, D. M. (2004). Respiratory chain enzyme analysis in muscle and liver. Mitochondrion 4, 363-375. doi: 10.1016/ j.mito.2004.07.003
Wong, L. J., Brunetti-Pierri, N., Zhang, Q., Yazigi, N., Bove, K. E., Dahms, B. B., et al. (2007). Mutations in the MPV17 gene are responsible for rapidly progressive liver failure in infancy. Hepatology 46, 1218-1227. doi: 10.1002/ hep. 21799

Xiao, M., Yang, H., Xu, W., Ma, S., Lin, H., Zhu, H., et al. (2012). Inhibition of alpha-KG-dependent histone and DNA demethylases by fumarate and succinate that are accumulated in mutations of FH and SDH tumor suppressors. Genes Dev. 26, 1326-1338. doi: 10.1101/gad.191056.112

Zavala, G. (2006). Runting Stunting Syndrome (RSS) in Broilers. In Vivo Stud.

Zeviani, M., Bonilla, E., DeVivo, D. C., and DiMauro, S. (1989). Mitochondrial diseases. Neurol. Clin. 7, 123-156. doi: 10.1053/beog.2002.0315

Zhang, L., Li, Y., Xie, X., Xu, H., Xu, Z., Ma, J., et al. (2015). A Systematic Analysis on mRNA and MicroRNA expression in runting and stunting chickens. PloS One 10, e0127342. doi: 10.1371/journal.pone.0127342

Zorov, D. B., Juhaszova, M., and Sollott, S. J. (2014). Mitochondrial reactive oxygen species (ROS) and ROS-induced ROS release. Physiol. Rev. 94, 909950. doi: 10.1152/physrev.00026.2013

Conflict of Interest: The authors declare that the research was conducted in the absence of any commercial or financial relationships that could be construed as a potential conflict of interest.

Copyright $\odot 2020 \mathrm{Li}, \mathrm{Hu}$, Luo, Hu, Luo, Zhao, Gan, Li, Shi, Nie, Zhang and Zhang. This is an open-access article distributed under the terms of the Creative Commons Attribution License (CC BY). The use, distribution or reproduction in other forums is permitted, provided the original author(s) and the copyright owner(s) are credited and that the original publication in this journal is cited, in accordance with accepted academic practice. No use, distribution or reproduction is permitted which does not comply with these terms. 